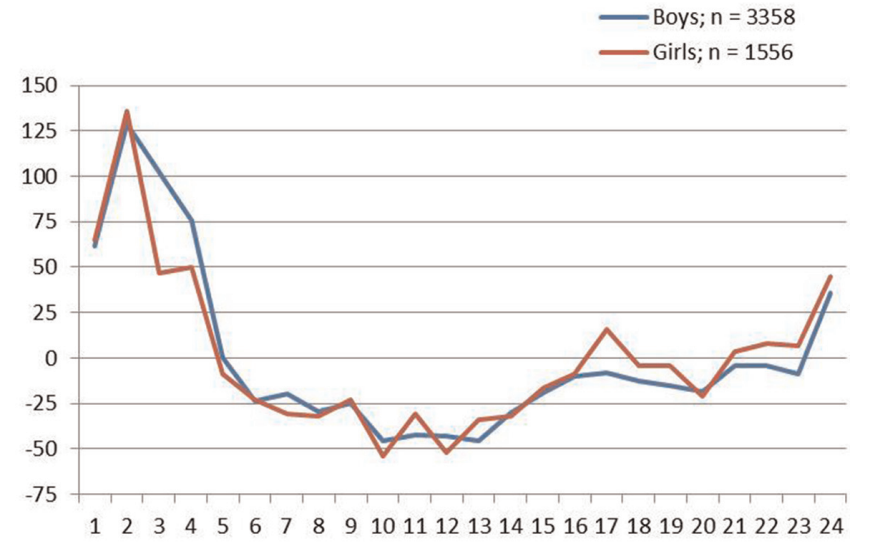

Abstract P0-0208 Figure 2 Hourly percentage variation in emergency department calls for croup in Vinnytsya, Ukraine in 19952008 (0 represents the sum hourly variations)

\section{PO-0209 CLINICAL FEATURE OF HOSPITALISED CHILDREN WITH INFLUENZA INFECTION FOR NINE YEARS}

H Uryu, R Yamada, M Kubota, K Okuma, M Tanaka, Y Okuma, J Yamanaka, N Sato, T Matsushita. Pediatrics, National Center for Global Health and Medicine, Shinjuku-Ku, Japan

\subsection{6/archdischild-2014-307384.864}

The relation between influenza subtype and age, clinical symptoms, diagnosis, and clinical course was reviewed in hospitalised children in winter season annually since 2005 to 2014 respectively. The diagnosis of influenza was made by rapid diagnostic test kit or PCR test. The clinical features in each type of influenza were evaluated according to respiratory (R: pneumonia), neurological ( $\mathrm{N}$; seizure, abnormal behaviour), encephalopathy (E), and other symptoms (O;persistent fever, dehydration, gastroenteritis and others). The differential test for 2009 H1N1 was not performed in 2013-14 season.

Results 292 (180 boys, and 112 girls) cases were admitted due to influenza antigen positive disease in this study period. Seasonal type A was 128 (R; 45, N44, E;5, O;33), 2009 H1N1 was 95 (R:55, N;23, E;3, O;14), and type B was 69 (R;27, N;21, $\mathrm{E} ; 2, \mathrm{O} ; 19)$ cases. $2009 \mathrm{H} 1 \mathrm{~N} 1$ virus was detected only in200910 and $2010-11$ seasons. The age distribution $(0 \mathrm{y} / 1-5 \mathrm{y} / 6-10 \mathrm{y} /$ $11 \mathrm{y}-$ ) of these children was 26/64/32/6 in seasonal A, 10/24/40/ 21 in $2009 \mathrm{H} 1 \mathrm{~N} 1$, and 1/33/29/6in type B. All children recovered and discharged without major complication. In respiratory disorder, percutaneous Oxygen saturation at admission was lower in $2009 \mathrm{H} 1 \mathrm{~N} 1$ (median 88\%) than seasonal A (96) or B (98), but total admission period was not longer.

Conclusion Rapid diagnostic kit is useful to know the origin of fever when patient should be admitted. The results support to understand the clinical picture of age related symptoms.

\section{PO-0210 FEATURES OF HCV INFECTION IN CHILDREN FROM A HEPATOLOGY DEPARTMENT IN SOUTH-EASTERN EUROPE}

D Pacurar, ID Nicolaescu, R Nicolaescu, RM Vlad, I Andronie, A Moraru, G Lesanu, D Oraseanu. Department of Pediatrics, "Grigore Alexandrescu" Emergency Children's Hospital, Bucharest, Romania

10.1136/archdischild-2014-307384.865
Background and aims Hepatitis C virus (HCV) infection is cause of significant morbidity and mortality, especially in children. The study aimed to describe the epidemiological characteristics of children infected with HCV diagnosed in "Grigore Alexandrescu" Children's Hospital, Bucharest.

Methods We reviewed the medical records of HCV infected children diagnosed between January 1991 and December 2012 and analysed demographic data, viral serology and route of infection.

Results We identified 58 cases, all diagnosed by detection of anti-HCV antibodies. $47.3 \%$ of the patients came from Bucharest, the rest residing in neighbouring districts. They were equally distributed by means of sex $(52.6 \%$ boys $)$ and age groups. Regarding alleged route of transmission: $43.7 \%$ had infected mothers (perinatal infection), $22.8 \%$ had a history of parenteral procedures (surgical intervention, blood transfusions, iv drug abuse, tattoos). $69.5 \%$ underwent evaluation for fibrosis, by either hepatic biopsy or fibroscan: $85 \%$ had a low fibrosis score $(<3) .58 \%$ of the patients had minimal cytolysis, with ALT less than 1.5 fold the normal value. Regarding viremia, $40 \%$ of the patients had less than 100000 copies and only $1.75 \%$ over 10 millions. No correlation was found between the level of transaminases and the viraemia or fibrosis scores on hepatic biopsy.

Conclusions HCV infection in children is a public health issue. Materno-fetal transmission is the main route of infection followed by transfusions and other parenteral routes. We predict that a thorough pregnancy monitorization and an accurate blood donor screening will significantly reduce the number of infected children in our country.

\section{PO-0211 EVALUATION OF PCR/ESI-MS PLATFORM TO IDENTIFY RESPIRATORY VIRUS FROM NASAL PHARYNGEAL ASPIRATES}

J Xu, M Xu, L Su. Department of Clinical Laboratory, Children's Hospital of Fudan University, Shanghai, China

\subsection{6/archdischild-2014-307384.866}

Background and aims Acute respiratory tract infections are a major cause of morbidity and mortality worldwide, particularly in infants and young children. High-throughput, accurate, broad etiologic diagnosis tools were critical for effective epidemic control. In this study, the diagnostic capacities of an Ibis platform based on PCR/ESI-MS assay were evaluated on clinical samples. Methods A total of 120 NPAs were collected from 120 children ( $<5$ years old) hospitalised with lower respiratory infections between November 2010 and October 2011. The respiratory virus detection assay was performed with PCR/ESI-MS assay and DFA assay respectively. The all discordant PCR/ESI-MS and DFA results were resolved with RT-PCR plus sequencing.

Results The overall agreement for PCR/ESI-MS and DFA was 98.3\% (118/120). Compared to the results from DFA, the sensitivity and specificity of the PCR/ESI-MS assay were $100 \%$ and 97.5\%, respectively. The PCR/ESI-MS assay also detected more multi-viruses infection and demonstrated more detailed types information than DFA. Among the 12 original specimens with disagreement results between PCR/ESI-MS and DFA, 9 have confirmed PCR/ESI-MS results.

Conclusion This assay is a high-throughput, sensitive, specific and promising method to detect and subtype the conventional viruses for respiratory tract infections, and allowed rapid identification of mixed pathogens. 